\title{
A ciência da vulnerabilidade: modelos, métodos e indicadores
}

Vulnerability Science: Models, Methods, and Indicators

La science de la vulnérabilité: modèles, méthodes et indicateurs

Susan L. Cutter

Tradutor: Victor Ferreira

\section{(2) OpenEdition}

\section{Journals}

Edição electrónica

URL: http://journals.openedition.org/rccs/165

DOI: $10.4000 /$ rccs. 165

ISSN: 2182-7435

Editora

Centro de Estudos Sociais da Universidade de Coimbra

\section{Edição impressa}

Data de publição: 1 Junho 2011

Paginação: 59-69

ISSN: 0254-1106

\section{Refêrencia eletrónica}

Susan L. Cutter, «A ciência da vulnerabilidade: modelos, métodos e indicadores », Revista Crítica de Ciências Sociais [Online], 93 | 2011, colocado online no dia 01 outubro 2012, criado a 19 abril 2019. URL : http://journals.openedition.org/rccs/165 ; DOI : 10.4000/rccs.165

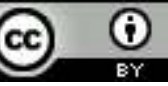




\title{
A ciência da vulnerabilidade: Modelos, métodos e indicadores
}

\begin{abstract}
A ciência da vulnerabilidade consiste na integração multidisciplinar das ciências sociais, das ciências naturais e da engenharia na compreensão das circunstâncias que colocam as populações e os locais em risco devido aos perigos, e dos factores que aumentam ou reduzem a capacidade de resposta e de recuperação das populações, dos sistemas físicos ou das infraestruturas em relação a ameaças ambientais. A integração das diversas perspectivas requer análises baseadas nos locais com recurso a ferramentas e tecnologias geoespaciais. Este artigo descreve os instrumentos e os métodos para medir e cartografar a exposição ao risco (chamada vulnerabilidade física), a medição e a cartografia da propensão das populações para os riscos (designada vulnerabilidade social) e a intersecção dos dois para criar as análises baseadas nos locais. A intersecção da vulnerabilidade física e da vulnerabilidade social cria a paisagem dos riscos (hazardscape) que, por sua vez, ajuda os investigadores a compreender os impactos diferenciados dos riscos e dos desastres nos locais e nos respectivos habitantes.
\end{abstract}

Palavras-chave: desastres naturais; factores de risco; furacão Katrina; sismos; sociologia do risco; vulnerabilidade social.

\section{Introdução}

Por todo o mundo encontramos vários exemplos da forma como um único acontecimento perigoso, como um furacão ou um sismo, produz impactos muito diferentes. Considere-se o caso do Furacão Katrina ao longo da costa dos Estados Unidos da América (EUA) no Golfo do México. Em Nova Orleães, no estado da Luisiana, o furacão produziu efeitos muito diferentes dos que provocou em Gulfport, no estado vizinho do Mississípi. Para além das diferenças entre as causas (a ruptura de diques e a transposição destes em Nova Orleães devido à ondulação do mar provocada pela tempestade, e na costa do estado do Mississípi a ondulação e o vento), houve uma diferenciação significativa nos impactos nas comunidades atingidas. Embora o furacão tenha afectado toda a população na região, houve comunidades que foram mais afectadas do que outras, facto que se ficou a dever às condições 
sociais subjacentes, que as tornaram mais ou menos vulneráveis ao acontecimento. Outros exemplos de impactos diferenciados são o sismo de 2010 no Haiti (magnitude 7,0) e o sismo de maiores proporções em Maule, no Chile (magnitude 8,8), quase um mês mais tarde. O número de vítimas no Haiti foi extremamente elevado, tendo-se estimado entre 200000 e 250000 mortos (The New York Times, 2010). No Chile, a estimativa aponta para cerca de 500 vítimas mortais, um número significativamente mais baixo, apesar da magnitude superior do sismo. Uma explicação possível para esta disparidade ao nível do impacto humano nos dois países está relacionada com a vulnerabilidade do Haiti e da sua população em comparação com o Chile e a população chilena.

\section{O que é a ciência da vulnerabilidade?}

A vulnerabilidade, numa definição lata, é o potencial para a perda. A vulnerabilidade inclui quer elementos de exposição ao risco (as circunstâncias que colocam as pessoas e as localidades em risco perante um determinado perigo), quer de propensão (as circunstâncias que aumentam ou reduzem a capacidade da população, da infraestrutura ou dos sistemas físicos para responder a e recuperar de ameaças ambientais). Embora estes conceitos tenham sido já muito discutidos na literatura, estas definições tão simples e genéricas apreendem a essência da vulnerabilidade (Cutter, 1996; Turner et al., 2003; Wisner et al., 2004; Adger, 2006; Birkmann, 2006). A ciência da vulnerabilidade fornece a base empírica para a elaboração de políticas de redução de riscos através do desenvolvimento de métodos e métricas para analisar a vulnerabilidade societal aos riscos ambientais e aos acontecimentos extremos (Cutter, 2003). Em particular, a ciência da vulnerabilidade procura analisar os factores que influenciam as capacidades locais (definidas aqui como subnacionais) na preparação para, resposta a e recuperação de desastres, examinando de forma comparativa os vários padrões daí resultantes.

Para isso é necessária uma abordagem integradora, que procure explicar as complexas interacções entre sistemas sociais, naturais e artificiais. Embora seja importante entender os sistemas e processos naturais que dão origem aos riscos, não é possível compreender completamente o impacto de tais processos ou acontecimentos naturais, a menos que se examine a forma como esses sistemas interagem com a sociedade. Por exemplo, os incêndios são processos naturais e, em ecossistemas de chaparral como os que se encontram nas regiões com clima mediterrânico, o ecossistema necessita de fogos periódicos de modo a poder sobreviver. Só quando estes incêndios florestais ocorrem perto de áreas povoadas se tornam 
algo mais do que simples processos naturais, uma vez que o potencial de perda aumenta nessas áreas urbano-florestais. De igual modo, a ciência da vulnerabilidade examina as interacções entre sistemas sociais e sistemas e estruturas artificiais, como barragens, diques, estruturas de protecção costeira ou redes urbanas e de transporte. A vulnerabilidade tem origem no potencial de perda e de impacto negativo que estes sistemas e/ou estruturas têm nas pessoas, ao falhar. Outro princípio fundamental da ciência da vulnerabilidade é o requisito do conhecimento geoespacial e da investigação com base nos locais. Todos os desastres são locais e as respostas imediatas também o são.

A ciência da vulnerabilidade ajuda a perceber como o mesmo acontecimento pode produzir impactos muito diferentes nas zonas envolventes, como foi ilustrado pelos exemplos apresentados na introdução. Para além disso, fornece instrumentos que permitem investigar a forma como os factores societais interferem na natureza e no ambiente construído, redistribuindo o risco anterior a um acontecimento e os prejuízos após o mesmo. A comparação entre lugares requer métricas consistentes, daí que o conceito de medição seja crucial na ciência da vulnerabilidade. De facto, a capacidade sistemática para comparar uma localidade com outra em termos da sua vulnerabilidade é uma componente fundamental da ciência da vulnerabilidade.

As condições naturais ou ambientais que ajudam a compreender a exposição ao risco têm geralmente por base informação proveniente das ciências naturais. A análise do ambiente construído ou das infraestruturas está adstrita às ciências da engenharia, incluindo medições das infraestruturas críticas (oleodutos, redes de transporte, sistemas de comunicação), assim como do edificado (residencial, comercial, industrial, institucional). Por último, é necessário efectuar a medição das condições sociais, geralmente com recurso a dados socioeconómicos e a outros dados demográficos. A unidade de medida pode ser individual (uma pessoa, um agregado familiar, uma estrutura), um grupo (grupos sociais, como estudantes universitários, bairros e infraestruturas), ou uma entidade espacial (um município, uma freguesia ou outra unidade administrativa) em que o ambiente social e construído e os dados físicos estejam integrados.

\section{Cartografar a exposição ao risco}

A maioria da investigação relacionada com os perigos concentra-se na cartografia dos sistemas físicos e em delinear o risco associado aos perigos. Neste sentido, verifica-se o recurso a Sistemas de Informação Geográfica (SIG) para cartografar planícies aluviais (e o perigo de inundação), zonas de 
inundação costeira, perigos de deslizamento de terras, áreas sísmicas, etc. Nos EUA existe um número de modelos diferentes que efectuam este tipo de análises de exposição espacial para riscos particulares, como os de sismos, cheias e ventos ciclónicos (HAZUS) ou de inundações provocadas por tempestades nas zonas costeiras (SLOSH) ou de erosão costeira (Thieler e Hammar-Klose, 1999; Wald et al., 2003; Merz et al., 2007). Há cada vez mais modelos concebidos para examinar a delineação espacial das zonas de inundação provocada pela subida do nível do mar (Titus e Richman, 2001; Wu et al., 2002).

A delimitação das zonas de exposição é feita em qualquer escala e normalmente recorre a tecnologias com base nos SIG (Jensen e Hodgson, 2006; Cutter et al., 2007). Frequentemente representadas por uma linha ou por um polígono no mapa da área em estudo, as zonas de exposição podem ser agregadas a uma unidade política ou administrativa, como um município ou um distrito, para efeitos de comparação numa área geográfica mais vasta. Há muitos exemplos na literatura publicada, nomeadamente em revistas como a Natural Hazards, a Applied Geography, a Earthquake Spectra ou a Natural Hazards Review. Também estão disponíveis estudos comparativos transnacionais, como os que são usados no Environmental Vulnerability Index da SOPAC (www.vulnerabilityindex.net/EVI_Background. htm) ou o Prevalent Vulnerability Index (www.iadb.org/exr/disaster/pvi. $\mathrm{cfm}$ ?language $=$ en\&parid $=4$ ), publicado pelo Banco Interamericano de Desenvolvimento (BID).

\section{Medir a propensão ao risco}

A vulnerabilidade social é o conceito que traduz a propensão da população para os impactos negativos dos perigos e dos desastres (Cutter et al., 2003; Laska e Morrow, 2006). Ela identifica as características da população que aumentam ou diminuem a sua capacidade de preparação para, resposta a e recuperação de um acontecimento perigoso ou de um desastre. A vulnerabilidade social ajuda também a compreender a distribuição dos riscos e das perdas potenciais, ou seja, a relação existente entre as populações vulneráveis e os ambientes naturais vulneráveis. Existe um grande número de estudos sobre populações vulneráveis, muitos dos quais se baseiam em inquéritos e estudos empíricos pós-desastre realizados no âmbito das ciências sociais. Algumas das características sociais que influenciam a vulnerabilidade social encontram-se na Tabela 1.

Apesar destes conhecimentos, resultantes de vários estudos de caso, existem poucos esforços sistemáticos para quantificar a vulnerabilidade social de lugares específicos. Um desses esforços consubstancia-se no 
TABELA 1 - Exemplos de características que influenciam a vulnerabilidade social

\begin{tabular}{|c|c|c|c|}
\hline Conceito & Fundamentação & Variável & $\begin{array}{c}\text { Natureza } \\
\text { da influência }\end{array}$ \\
\hline $\begin{array}{l}\text { Populações com } \\
\text { necessidades } \\
\text { especiais }\end{array}$ & $\begin{array}{l}\text { Difíceis de identificar (doentes } \\
\text { ou temporárias), muitas vezes } \\
\text { invisíveis nas comunidades }\end{array}$ & $\begin{array}{l}\text { População sem-abrigo } \\
\text { Residentes em lares }\end{array}$ & aumenta \\
\hline Idade & $\begin{array}{l}\text { Afecta a mobilidade; requer } \\
\text { cuidados especiais; maior } \\
\text { susceptibilidade para se magoar }\end{array}$ & $\begin{array}{l}\text { Idosos } \\
\text { Crianças }\end{array}$ & $\begin{array}{l}\text { aumenta } \\
\text { aumenta }\end{array}$ \\
\hline $\begin{array}{l}\text { Estatuto } \\
\text { socioeconómico }\end{array}$ & $\begin{array}{l}\text { Capacidade de absorver danos e } \\
\text { de recuperar; mais bens materiais } \\
\text { a perder }\end{array}$ & $\begin{array}{l}\text { Ricos } \\
\text { Pobres }\end{array}$ & $\begin{array}{l}\text { diminui } \\
\text { aumenta }\end{array}$ \\
\hline Raça e etnia & $\begin{array}{l}\text { Barreiras linguísticas e culturais; } \\
\text { falta de acesso a recursos pós- } \\
\text {-desastre; tendência para ocupar } \\
\text { zonas de perigosidade elevada }\end{array}$ & Hispânicos (nos EUA) & aumenta \\
\hline Sexo & $\begin{array}{l}\text { Empregos com altas taxas de } \\
\text { feminização podem ser afectados; } \\
\text { salários mais baixos; tarefas de } \\
\text { prestação de cuidados }\end{array}$ & Mulheres & aumenta \\
\hline $\begin{array}{l}\text { Tipo de habitação } \\
\text { e título de } \\
\text { propriedade }\end{array}$ & $\begin{array}{l}\text { Com frequência, os inquilinos } \\
\text { não têm seguro nem investem na } \\
\text { comunidade; tipo de habitação e } \\
\text { construção }\end{array}$ & $\begin{array}{l}\text { Inquilinos } \\
\text { Habitações móveis }\end{array}$ & $\begin{array}{l}\text { aumenta } \\
\text { aumenta }\end{array}$ \\
\hline
\end{tabular}

Fonte: Heinz Center, 2002; Cutter et al., 2003.

Social Vulnerability Index - SoVI ${ }^{\mathrm{TM}}$ (Índice de Vulnerabilidade Social). O SoVI é uma avaliação quantitativa das características que influenciam a vulnerabilidade social aos riscos (pré-acontecimentos) e facilita a comparação entre unidades geográficas (distritos, secções censitárias) em termos dos seus níveis relativos de vulnerabilidade social. Os perfis socioeconómicos são gerados a partir da informação dos censos e submetidos a um procedimento estatístico para reduzir o número de variáveis a um conjunto menor de factores que descrevem a vulnerabilidade (Cutter et al., 2003). Desta forma, o SoVI consegue apreender a natureza multidimensional da vulnerabilidade social. Por exemplo, a raça ou a etnia por si só não indicam necessariamente populações vulneráveis mas, quando combinadas com a idade e o sexo, podem torná-las mais sensíveis aos riscos (por exemplo, as mulheres idosas afro-americanas). ${ }^{1}$ Em grande medida, o SoVI é mais um algoritmo para desenvolver perfis de vulnerabilidade social do que uma ferramenta

${ }^{1}$ Para mais informações sobre a construção do SoVI, ver http://sovius.org. 
propriamente dita. Foi replicado nos EUA com censos mais antigos (Cutter e Finch, 2008), aplicado em escalas menores, como unidades censitárias e unidades territoriais (Schmidtlein et al., 2008), e adaptado a outros países, como a Noruega (www.svt.ntnu.no/geo/Doklager/Projects/SoVI_Norway. pdf), o Vietname (Nhuan et al., 2009), e Barbados e St. Vincent (Boruff e Cutter, 2007).

A expressão geográfica do Índice de Vulnerabilidade Social é um mapa que representa os extremos da distribuição estatística dos resultados do índice (normalmente superior ou inferior a um desvio-padrão da média). Deste modo, o mapa ilustra as áreas que têm níveis relativamente elevados de vulnerabilidade social, assim como as que têm níveis relativamente baixos. Por exemplo, a Figura 1 apresenta o mapa da vulnerabilidade social dos EUA, em 2000. O nível mais baixo de concentração de vulnerabilidade social encontra-se ao longo da faixa costeira oriental, de Washington D.C. a Nova Iorque e na área metropolitana de Boston. Trata-se de uma região rica, sem níveis extremos de envelhecimento e de feminização da população. Os condados costeiros da Califórnia também registam níveis relativamente baixos de vulnerabilidade social. Por outro lado, os condados representados a vermelho caracterizam-se por uma combinação de factores que resultam em elevados níveis de vulnerabilidade social. Por exemplo, o troço inferior do Rio Mississípi atravessa uma região historicamente pobre, de população maioritariamente afro-americana. Nestes condados há pouco emprego, baixos níveis de escolaridade, acesso limitado aos serviços de saúde pela sua natureza rural e um número significativo de agregados familiares chefiados por mulheres. Todos estes factores contribuem para níveis elevados de vulnerabilidade.

\section{A intersecção da exposição e da propensão}

Ao representar a vulnerabilidade local é importante considerar não apenas um dos elementos da vulnerabilidade - físico ou social -, mas a sua intersecção. Em alguns lugares, a vulnerabilidade física pode ser bastante elevada (por exemplo, nas zonas costeiras), mas se a população residente for rica, com recursos consideráveis para a preparação para e resposta a desastres (o que equivale a menor vulnerabilidade social), a comunidade será capaz de recuperar rapidamente. Se, por outro lado, a comunidade costeira vizinha (com o mesmo nível de exposição física) apresentar características sociais diferentes (residentes pobres, idosos e pertencentes a minorias), então essa comunidade irá demorar mais tempo a recuperar, uma vez que a capacidade dos seus habitantes para absorver as perdas e recuperar é também mais limitada. 


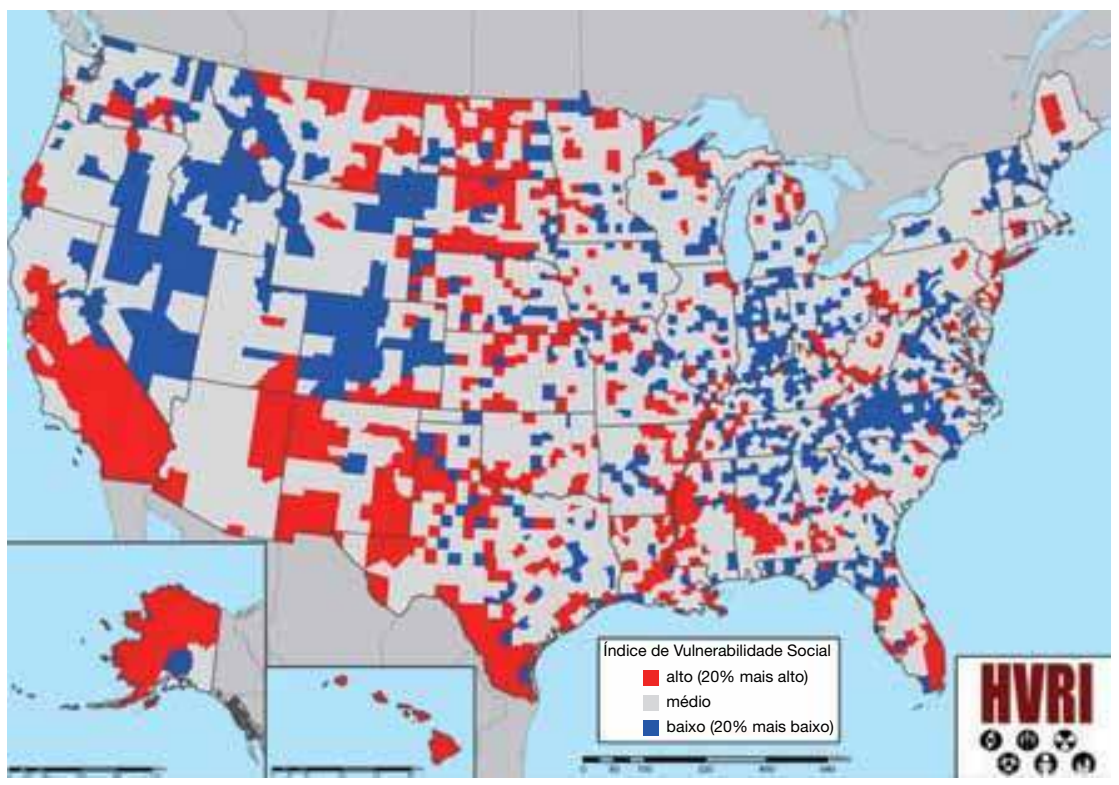

Fonte: http://webra.cas.sc.edu/hvri/image/figure/SoVI2000.png

Existem diversas formas de calcular a intersecção da vulnerabilidade física com a social, sendo que a cartografia espacial é a mais vantajosa (Cutter et al., 2000; O'Brien et al., 2004; Zahran et al., 2008). Por exemplo, Burton e Cutter (2008) analisaram o risco de cheia na área do Delta Sacramento-San Joaquin, na Califórnia. Cartografaram as zonas de cheia para períodos de retorno de 100 e 500 anos (com base em dados modelados do software HAZUS da FEMA, Federal Emergency Management Agency - Agência Federal de Gestão de Emergências), calculando posteriormente um índice de vulnerabilidade social para a área em estudo. Recorrendo aos SIG, a intersecção dos dois reflectiu a vulnerabilidade total ao risco de cheias. A vantagem deste tipo de procedimento é o facto de permitir identificar o principal factor que determina essa vulnerabilidade - a exposição ao risco, a propensão, ou ambos os factores em igual medida. Outra abordagem é o recurso à cartografia bivariada, em que a exposição ao risco e a vulnerabilidade social são representadas num mapa coropleto com três categorias (elevada, média e baixa). Quando cartografadas em simultâneo, a exposição ao risco e a vulnerabilidade social dão origem a uma matriz de 9 células (ver Figura 2). Neste exemplo, as comunidades na parte superior direita (elevada exposição ao risco, elevada vulnerabilidade social) seriam as mais vulneráveis 
de todas. No entanto, as comunidades com elevada exposição ao risco e vulnerabilidade social média ou vice-versa também seriam mais vulneráveis, mas o factor determinante seria ligeiramente diferente. Um bom exemplo do uso de cartografia bivariada para a avaliação da vulnerabilidade pode ser visto na ferramenta da Oxfam de vulnerabilidade e alterações climáticas no Sudeste dos EUA (http://adapt.oxfamamerica.org). Outro exemplo é a cartografia da comparação dos níveis de inundação na cidade de Nova Orleães com a vulnerabilidade social subjacente (Finch et al., 2010).

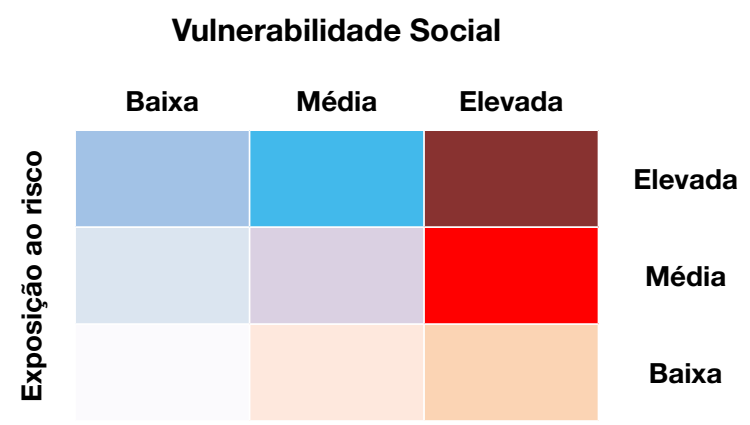

FIGURA 2 - Exemplo de cartografia bivariada

\section{Conclusão}

Este artigo ilustra a forma como a exposição ao risco e a vulnerabilidade social interagem para criar as paisagens de riscos (bazardscapes). A ciência da vulnerabilidade fornece a base para a construção de métricas para a vulnerabilidade, que incluem o cálculo de índices para medição da vulnerabilidade social, assim como modelos geoespaciais para a intersecção dos processos sociais e físicos. A importância da ciência da vulnerabilidade advém do facto de que a estratégia de gestão do risco de bitola única ignora a realidade da desigualdade social e dos diferentes custos sociais que os riscos acarretam. A ciência da vulnerabilidade aos riscos lembra-nos que o espaço e o local têm importância e proporciona-nos as ferramentas e as técnicas necessárias para demonstrar empiricamente esse princípio.

Tradução de

Victor Ferreira 


\section{Referências bibliográficas}

Adger, Neil (2006), "Vulnerability", Global Environmental Change, 16, 268-281.

Birkmann, Jörn. (org.) (2006), Measuring Vulnerability to Natural Hazards: Towards disaster resilient societies. Tóquio: United Nations University Press.

Boruff, Bryan; Cutter, Susan (2007), "The Environmental Vulnerability of Caribbean Island Nations", The Geographical Review, 91(1), 24-45.

Burton, Christopher; Cutter, Susan (2008), "Levee Failures and Social Vulnerability in the Sacramento-San Joaquin Delta Area, California", Natural Hazards Review, 9(3), 136-149.

Cutter, Susan (1996), "Vulnerability in Environmental Hazards", Progress in Human Geography, 20(4), 529-539.

Cutter, Susan (2003), "The Vulnerability of Science and the Science of Vulnerability”, Annals of the Association of American Geographers, 93(1), 1-12.

Cutter, Susan; Mitchell, Jerry; Scott, Michael (2000), "Revealing the Vulnerability of People and Places: A case study of Georgetown County, South Carolina”, Annals of the Association of American Geographers, 90(4), 713-737. Consultado em http:// webra.cas.sc.edu/hvri/docs/gtown.pdf.

Cutter, Susan; Boruff, Bryan; Shirley, Lynn (2003), "Social Vulnerability to Environmental Hazards”, Social Science Quarterly, 84(1), 242-261 Consultado em http:// webra.cas.sc.edu/hvri/pubs/2003_SocialVulnerabilitytoEnvironmentalHazards.pdf.

Cutter, Susan; Emrich, Christopher; Adams, Beverley; Huyck, Charles; Eguchi, Ronald (2007), "New Information Technologies in Emergency Management", in William Waugh e Kathleen Tierney (orgs.), Emergency Management: Principles and practice for local government. Washington DC: ICMA Press, 279-298 [2. ${ }^{a}$ ed.].

Cutter, Susan; Finch, Christina (2008), "Temporal and Spatial Changes in Social Vulnerability to Natural Hazards", Proceedings of the National Academy of Sciences, US (PNAS), 105(7), 2301-2306. Consultado em http://www.pnas.org/ content/105/7/2301.full.pdf+html.

Finch, Christina; Emrich, Christopher; Cutter, Susan (2010), "Disaster Disparities and Differential Recovery in New Orleans”, Population and Environment, 31(4), 179-202. Heinz Center (2002), Human Links to Coastal Disasters. Washington D.C.: The H. John Heinz III Center for Science, Economics and the Environment.

Jensen, John; Hodgson, Michael (2006), "Remote Sensing of Natural and Man-Made Hazards and Disasters", in Merril Ridd e James Hipple (orgs.), Remote Sensing of Human Settlements, Manual of Remote Sensing, Vol. 5. Bethesda, MA: American Society of Photogrammetry and Remote Sensing, 401-429 [3. a ed.].

Laska, Shirley; Morrow, Betty (2006), "Social Vulnerabilities and Hurricane Katrina: An unnatural disaster in New Orleans”, Marine Technology Society Journal, 40(4), 16-26.

Merz, Bruno; Thieken, Annegret; Gocht, Martin (2007), "Flood Risk Mapping at the Local Scale: Concepts and challenges”, in Selina Begum, Marcel Stive e Jim 
Hall (orgs.), Flood Risk Management in Europe. Innovation in Policy and Practice. Dordrecht: Springer, 231-251.

Nhuan, Mai; Ngoc, Nguyen; Huong, Nghiem; Hue, Nguyen; Tue, Nguyen; Ngoc, Pham (2009), "Assessment of Vietnam Coastal Wetland Vulnerability for Sustainable Use (Case Study in Xuanthuy Ramsar Site, Vietnam)", J. Wetlands Ecology, 2, 1-16.

O’Brien, Karen; Leichenko, Robin; Kelkarc, Ulka; Venemad, Henry; Aandahla, Guro; Tompkinsa, Heather; Javedc, Akram; Bhadwalc, Suruch; Bargd, Stephan; Nygaarda, Lynn; West, Jennifer (2004), "Mapping Vulnerability to Multiple Stressors: Climate change and globalization in India", Global Environmental Change, 14, 303-313. Consultado em http://www.c-ciarn.uoguelph.ca/documents/OBrien_2004.pdf.

Schmidtlein, Mathew; Deutsch, Roland; Piegorsch, Walter; Cutter, Susan (2008), "Building Indexes of Vulnerability: A sensitivity analysis of the social vulnerability index”, Risk Analysis, 28(4), 1099-1114. Consultado em http://www.ihdp.unu.edu/ file/get/3596.pdf.

Thieler, Robert; Hammar-Klose, Erika (1999), National Assessment of Coastal Vulnerability to Future Sea-Level Rise: Preliminary results for the U.S. Atlantic Coast. U.S. Geological Survey, Open-File Report 99-593. Consultado em http://pubs.usgs.gov/ of/of99-593.

The New York Times (2010), Haiti Estimates of Quake Damage in Haiti Increase by Billions, 16 de Fevereiro. Consultado em http://www.nytimes.com/2010/02/17/ world/americas/17haiti.html? ref=haiti.

Titus, James; Richman, Charlie (2001), "Maps of Lands Vulnerable to Sea Level Rise: Modeled elevations along the US Atlantic and Gulf coasts", Climate Research, 18, 205-228. Consultado em http://www.epa.gov/climatechange/effects/downloads/ maps.pdf.

Turner, Billie; Kasperson, Roger; Matson, Pamela; McCarthy, James; Corell, Robert; Christensen, Lindsey; Eckley, Noelle; Kasperson, Jeanne; Luers, Amy; Martello, Marybeth; Polsky, Colin; Pulsipher, Alexander; Schiller, Andrew (2003), "A Framework for Vulnerability Analysis in Sustainability Science”, Proceedings of the National Academy of Sciences US (PNAS), 100, 8074-8079. Consultado em http://www.pnas. org/content/100/14/8074.full.pdf+html.

Wald, David; Wald, Lisa; Worden, Bruce; Goltz, Jim (2003), ShakeMap-A Tool for Earthquake Response, U.S. Geological Survey Fact Sheet 087-03. Consultado em http://pubs.usgs.gov/fs/fs-087-03.

Wisner, Ben; Blaikie, Piers; Cannon, Terry; Davis, Ian (2004), At Risk: Natural bazards, people's vulnerability and disasters. New York: Routledge.

Wu, Shuang-Ye; Yarnal, Brent; Fisher, Ann (2002), "Vulnerability of Coastal Communities to Sea Level Rise: A case study of Cape May County, New Jersey, USA", Climate Research, 22, 255-270. Consultado em http://www.int-res.com/ abstracts/cr/v22/n3/p255-270. 
Zahran, Sammy; Brody, Samuel; Peacock, Walter; Vedlitz, Arnold; Grover, Himanshu (2008), "Social Vulnerability and the Natural and Built Environment: A model of flood casualties in Texas”, Disasters, 32(4), 537-560. Consultado em http://www. texasemergingcommunities.org/resources. 